\begin{tabular}{|c|c|}
\hline Title & Curvilinear hybrid edge/hodal elements with triangular shape for guided-wave problems \\
\hline Author(s) & Koshiba, Masanori; Tsuji, Y asuhide \\
\hline Citation & $\begin{array}{l}\text { JOURNAL OF LIGHTWA VE TECHNOLOGY, 18(5), 737-743 } \\
\text { https://doi.org/10.1109/50.842091 }\end{array}$ \\
\hline Issue Date & $2000-05$ \\
\hline Doc URL & http:/hdl .handle.net/2115/5584 \\
\hline Rights & $\begin{array}{l}\text { ○2000 IEEE. Personal use of this material is permitted. However, permission to reprint/republish this material for } \\
\text { advertising or promotional purposes or for creating new collective works for resale or redistribution to servers or lists, } \\
\text { or to reuse any copyrighted component of this work in other works must be obtained from the IEEE." }\end{array}$ \\
\hline Type & article \\
\hline File Information & JLT18-5.pdf \\
\hline
\end{tabular}

Instructions for use 


\title{
Curvilinear Hybrid Edge/Nodal Elements with Triangular Shape for Guided-Wave Problems
}

\author{
Masanori Koshiba, Senior Member, IEEE, and Yasuhide Tsuji, Member, IEEE
}

\begin{abstract}
A unified approach using curvilinear hybrid edge/nodal elements with triangular shape is, for the first time, described for the study of guided-wave problems. Not only the lowest order (fundamental) but the higher order elements are systematically constructed. The advantage of curvilinear elements lies in the fact that they can model curved boundaries with more accuracy and lesser number of degrees of freedom than rectilinear elements. The vector basis functions derived here are also applicable to rectilinear cases. To show the validity and usefulness of the present approach, computed results are illustrated for rib waveguides with straight boundaries and circular waveguides with large refractive-index differences.
\end{abstract}

Index Terms-Curvilinear element, edge element, finite element method, full-wave analysis, guided-wave problem.

\section{INTRODUCTION}

$\mathbf{V}$ ARIOUS types of the finite element method have been developed for the full-vectorial analysis of guided-wave problems. Especially, the hybrid edge/nodal elements with triangular shape are very useful for imposing the continuity of the tangential components of the electric and magnetic fields and for eliminating the nonphysical, spurious solutions. The hybrid elements developed so far [1]-[3] are, however, only for the waveguides with straight, rectilinear boundaries.

Recently, Wang and Ida [4] have proposed curvilinear hexahedral and tetrahedral edge elements for solving cavity problems, and Antilla and Alexopoulos [5] have also proposed curvilinear brick edge elements for solving scattering problems. The advantage of curvilinear elements lies in the fact that they can model curved boundaries with more accuracy and lesser number of degrees of freedom than rectilinear elements, resulting in low computational cost. However, these three-dimensional elements belong to the lowest order, fundamental families, the convergence of which is, in general, not so fast. Furthermore, the curvilinear edge elements have not been specifically constructed for two-dimensional (2-D) problems, even though the lowest order ones.

In this paper, a unified approach using curvilinear hybrid edge/nodal elements with triangular shape is, for the first time, described for the study of guided-wave problems. After reviewing the earlier rectilinear edge elements [1]-[3], [6]-[8], not only the lowest order but the higher order curvilinear elements are systematically constructed. The validity and usefulness of the present approach are investigated in detail by calculating the propagation characteristics of rib waveguides

Manuscript received October 14, 1999; revised February 10, 2000 The authors are with the Division of Electronics and Information Engineering, Hokkaido University, Sapporo 060-8628, Japan.

Publisher Item Identifier S 0733-8724(00)03734-8.
TABLE I

DEFINITION OF SYMBOLS IN VECTORIAL WAVE EQUATION (1)

\begin{tabular}{ccc}
\hline$\phi$ & $p$ & $q$ \\
\hline $\boldsymbol{E}$ & 1 & $n^{2}$ \\
$\boldsymbol{H}$ & $1 / n^{2}$ & 1 \\
\hline
\end{tabular}

with straight boundaries and circular waveguides with large refractive-index difference.

\section{Vectorial WAVE EQUATION}

We consider a waveguide with arbitrary cross section $\Omega$ in the $x y$ (transverse) plane. With a time $(t)$ dependence of the form $\exp (j \omega t)$ being implied, from Maxwell's equations the following vectorial wave equation is derived:

$$
\nabla \times(p \nabla \times \boldsymbol{\phi})-k_{0}^{2} q \boldsymbol{\phi}=\mathbf{0}
$$

where $\omega$ is the angular frequency, $k_{0}$ is the free-space wavenumber, and $\phi, p$, and $q$ are given in Table I with $\mathbf{E}, \mathbf{H}$, and $n$ being, respectively, the electric field, the magnetic field, and the refractive index.

\section{Hybrid EDGE/NODAl ElEMENTS}

The electromagnetic fields have to be tangentially continuous across material interfaces. As is well-known, the tangential continuity in the guided-wave problem can be straightforwardly imposed in the hybrid edge/nodal elements, where the edge and the nodal elements are, respectively, employed for the transverse and the axial fields. Typical and well-established rectilinear hybrid elements with triangular shape are summarized in Fig. 1.

Fig. 1(a) shows the lowest order hybrid element [2], [6] which is composed of an edge element with three tangential variables, $\phi_{t 1}$ to $\phi_{t 3}$, based on constant tangential and linear normal $(\mathrm{CT} / \mathrm{LN})$ vector basis functions and a linear nodal (conventional Lagrange) element with three axial variables, $\phi_{z 1}$ to $\phi_{z 3}$. The tangential component of a particular CT/LN basis function is constant along one edge of the triangle and is zero along the other two edges, while the normal component is a linear function along all three edges [7].

Fig.1(b), (c), and (d) shows the higher order hybrid elements. For the axial fields, a quadratic nodal (conventional Lagrange) element with six axial variables, $\phi_{z 1}$ to $\phi_{z 6}$, is employed, while for the transverse fields, an edge element with six variables, $\phi_{t 1}$ to $\phi_{t 6}$, based on linear tangential and linear normal (LT/LN) basis functions [3], [8], or with eight variables, $\phi_{t 1}$ to $\phi_{t 8}$, based on linear tangential and quadratic normal (LT/QN) 


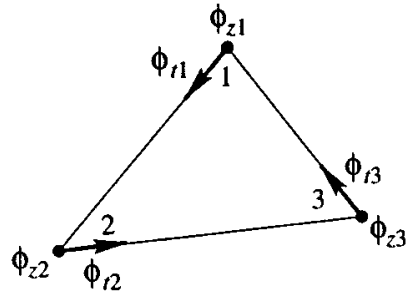

(a)

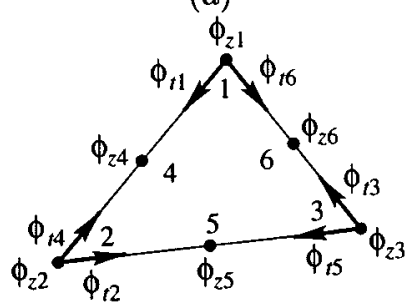

(b)

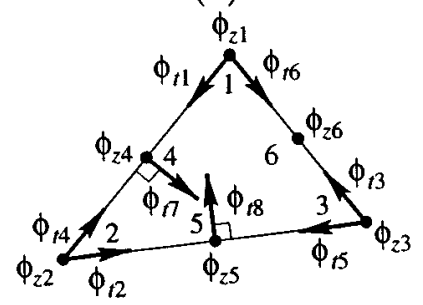

(c)

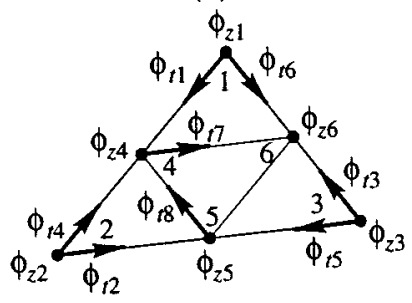

(d)

Fig. 1. Hybrid edge/nodal elements based on (a) constant tangential and linear normal (CT/LN), (b) linear tangential and linear normal (LT/LN), (c) linear tangential and quadratic normal (LT/QN-1), and (d) Nedelec's space-conforming linear tangential and quadratic normal (LT/QN-2) vector basis functions.

basis functions [1], [7] is employed. The LT/QN basis set is a combination of the six LT/LN functions and two additional quadratic functions. The six functions interpolate to a unity tangential component at the triangular edges. The other two functions each contribute a quadratic normal component to one (labeled as LT/QN-1) [1] or two (labeled as LT/QN-2) [7] of the three edges and have no tangential component along any of the edges. The LT/QN-1 and the LT/QN-2 basis functions, respectively, do not and do exactly satisfy the Nedelec constraints to eliminate some of nullspace degrees of freedom corresponding to irrotational, spurious solutions [7].

\section{CURVILINEAR HyBRID ELEMENTS}

Based on the rectilinear hybrid edge/nodal elements described in the previous section, the curvilinear counterparts are constructed as shown in Fig. 2. The interior variables $\phi_{t 7}$ and $\phi_{t 8}$ indicated by the solid and dashed arrows are associated with the LT/QN-1 and LT/QN-2 vector basis functions, respectively.

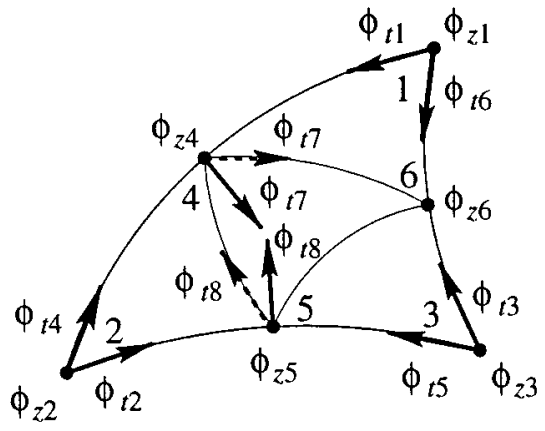

Fig. 2. Curvilinear hybrid edge/nodal element.

These interior variables are omitted in the LT/LN representation with six tangential variables, $\phi_{t 1}$ and $\phi_{t 6}$, and when using the $\mathrm{CT} / \mathrm{LN}$ representation, only the first three tangential variables, $\phi_{t 1}$ to $\phi_{t 3}$, remain.

In the curvilinear elements with triangular shape the Cartesian coordinates, $x$ and $y$, are, in general, approximated with quadratic polynomials using the local coordinates $L_{i}(i=1,2,3)[9],[10]$ as

$$
x=\sum_{j=1}^{6} N_{j} x_{j}, \quad y=\sum_{j=1}^{6} N_{j} y_{j}
$$

with

$$
\begin{aligned}
& N_{1}=L_{1}\left(2 L_{1}-1\right), \quad N_{2}=L_{2}\left(2 L_{2}-1\right) \\
& N_{3}=L_{3}\left(2 L_{3}-1\right), \quad N_{4}=4 L_{1} L_{2} \\
& N_{5}=4 L_{2} L_{3}, \quad N_{6}=4 L_{3} L_{1}
\end{aligned}
$$

where $x_{j}$ and $y_{j}$ are the Cartesian coordinates at the nodal point $j(j=1,2, \ldots, 6)$ within each element $e$ shown in Fig. 2. For the rectilinear elements with triangular shape, the local coordinates, $L_{1}, L_{2}$, and $L_{3}$, are called the area coordinates [9], [10].

Noting the relation among these local coordinates, $L_{1}+L_{2}+$ $L_{3}=1$, and selecting $L_{1}$ and $L_{2}$ as the independent variables, the transformation for differentiation is given by

$$
\begin{aligned}
{\left[\begin{array}{l}
\partial / \partial L_{1} \\
\partial / \partial L_{2}
\end{array}\right] } & =[J]\left[\begin{array}{l}
\partial / \partial x \\
\partial / \partial y
\end{array}\right] \\
& =\left[\begin{array}{ll}
J_{11} & J_{12} \\
J_{21} & J_{22}
\end{array}\right]\left[\begin{array}{l}
\partial / \partial x \\
\partial / \partial y
\end{array}\right]
\end{aligned}
$$

with

$$
\begin{aligned}
& J_{11}=\frac{\partial x}{\partial L_{1}}=\sum_{j=1}^{6} \frac{\partial N_{j}}{\partial L_{1}} x_{j} \\
& J_{12}=\frac{\partial y}{\partial L_{1}}=\sum_{j=1}^{6} \frac{\partial N_{j}}{\partial L_{1}} y_{j} \\
& J_{21}=\frac{\partial x}{\partial L_{2}}=\sum_{j=1}^{6} \frac{\partial N_{j}}{\partial L_{2}} x_{j} \\
& J_{22}=\frac{\partial y}{\partial L_{2}}=\sum_{j=1}^{6} \frac{\partial N_{j}}{\partial L_{2}} y_{j}
\end{aligned}
$$


TABLE II

VECTOR- AND SCALAR-BASED SHAPE FUNCTIONS

\begin{tabular}{|c|c|c|c|c|c|}
\hline edge & $\left\{\phi_{t}\right\}_{e}$ & $i_{x}\{U\}+i_{y}\{V\}$ & nodal & $\left\{\phi_{z}\right\}_{e}$ & $\{N\}$ \\
\hline $\mathrm{CT} / \mathrm{LN}$ & {$\left[\begin{array}{l}\phi_{t 1} \\
\phi_{t 2} \\
\phi_{t 3}\end{array}\right]$} & {$\left[\begin{array}{l}|J|_{1}\left|\nabla_{t} L_{3}\right|_{1}\left(L_{1} \nabla_{t} L_{2}-L_{2} \nabla_{t} L_{1}\right) \\
|J|_{2}\left|\nabla_{t} L_{1}\right|_{2}\left(L_{2} \nabla_{t} L_{3}-L_{3} \nabla_{t} L_{2}\right) \\
|J|_{3}\left|\nabla_{t} L_{2}\right|_{3}\left(L_{3} \nabla_{t} L_{1}-L_{1} \nabla_{t} L_{3}\right)\end{array}\right]$} & linear & {$\left[\begin{array}{l}\phi_{z 1} \\
\phi_{z 2} \\
\phi_{z 3}\end{array}\right]$} & {$\left[\begin{array}{l}L_{1} \\
L_{2} \\
L_{3}\end{array}\right]$} \\
\hline $\begin{array}{l}\mathrm{LT} / \mathrm{LN} \\
\mathrm{LT} / \mathrm{QN}-1 \\
\mathrm{LT} / \mathrm{QN}-2\end{array}$ & {$\left[\begin{array}{c}\phi_{t 1} \\
\phi_{t 2} \\
\phi_{t 3} \\
\phi_{t 4} \\
\phi_{t 5} \\
\phi_{t 6}\end{array}\right]$} & $\begin{array}{l}|J|_{1}\left|\nabla_{t} L_{3}\right|_{1}\left(L_{1} \nabla_{t} L_{2}\right) \\
|J|_{2}\left|\nabla_{t} L_{1}\right|_{2}\left(L_{2} \nabla_{t} L_{3}\right) \\
|J|_{3}\left|\nabla_{t} L_{2}\right|_{3}\left(L_{3} \nabla_{t} L_{1}\right) \\
|J|_{2}\left|\nabla_{t} L_{3}\right|_{2}\left(L_{2} \nabla_{t} L_{1}\right) \\
|J|_{3}\left|\nabla_{t} L_{1}\right|_{3}\left(L_{3} \nabla_{t} L_{2}\right) \\
|J|_{1}\left|\nabla_{t} L_{2}\right|_{1}\left(L_{1} \nabla_{t} L_{3}\right)\end{array}$ & quadratic & {$\left[\begin{array}{l}\phi_{z 1} \\
\phi_{z 2} \\
\phi_{z 3} \\
\phi_{z 4} \\
\phi_{z 5} \\
\phi_{z 6}\end{array}\right.$} & $\begin{array}{c}L_{1}\left(2 L_{1}-1\right) \\
L_{2}\left(2 L_{2}-1\right) \\
L_{3}\left(2 L_{3}-1\right) \\
4 L_{1} L_{2} \\
4 L_{2} L_{3} \\
4 L_{3} L_{1}\end{array}$ \\
\hline LT/QN-1 & $\begin{array}{l}\phi_{t 7} \\
\phi_{t 8}\end{array}$ & $\begin{array}{l}4 L_{1} L_{2} \nabla_{t} L_{3} /\left|\nabla_{t} L_{3}\right|_{4} \\
4 L_{2} L_{3} \nabla_{t} L_{1} /\left|\nabla_{t} L_{1}\right|_{5}\end{array}$ & & & \\
\hline $\mathrm{LT} / \mathrm{QN}-2$ & $\phi_{t 7}$ & $\begin{array}{l}4|J|_{4}\left|\nabla_{t} L_{1}\right|_{4}\left(L_{2} \nabla_{t} L_{3}-L_{3} \nabla_{t} L_{2}\right) \\
4|J|_{5}\left|\nabla_{t} L_{2}\right|_{5}\left(L_{3} \nabla_{t} L_{1}-L_{1} \nabla_{t} L_{3}\right)\end{array}$ & & & \\
\hline
\end{tabular}

where $[J]$ is the Jacobian matrix. The transformation relation for integration of a function $f(x, y)$ is given by

$$
\begin{aligned}
\iint_{e} f(x, y) d x d y \\
=\int_{0}^{1}\left[\int_{0}^{1-L_{1}} f\left(L_{1}, L_{2}, L_{3}\right)\left|J\left(L_{1}, L_{2}, L_{3}\right)\right| d L_{2}\right] d L_{1}
\end{aligned}
$$

where $|J|$ is the determinant of the Jacobian matrix and is called the Jacobian.

\section{Finite Element Discretization}

Dividing the waveguide cross section $\Omega$ into a number of curvilinear hybrid elements, we expand the transverse fields, $\phi_{x}$ and $\phi_{y}$, and the axial field $\phi_{z}$ in each element as

$$
\begin{aligned}
\phi= & \mathbf{i}_{x} \phi_{x}+\mathbf{i}_{y} \phi_{y}+\mathbf{i}_{z} \phi_{z} \\
= & \left.\mathbf{i}_{x}\{U\}^{T}+\mathbf{i}_{y}\{V\}^{T}\right)\left\{\phi_{t}\right\}_{e} \exp (-j \beta z) \\
& +\mathbf{i}_{z} j \beta\{N\}^{T}\left\{\phi_{z}\right\}_{e} \exp (-j \beta z)
\end{aligned}
$$

where $\mathbf{i}_{x}, \mathbf{i}_{y}$, and $\mathbf{i}_{z}$ are, respectively, the unit vectors in the $x, y$, and $z$ directions, $\left\{\phi_{t}\right\}_{e}$ and $\left\{\phi_{z}\right\}_{e}$ are, respectively, the edge- and nodal-variable vectors for each element, and $T$ denotes a transpose. The vector-based shape function for curvilinear edge elements, $\mathbf{i}_{x}\{U\}+\mathbf{i}_{y}\{V\}$, and the ordinary scalarbased shape function for curvilinear nodal elements, $\{N\}$, are given in Table II, where $\nabla_{t}=\mathbf{i}_{x} \partial / \partial x+\mathbf{i}_{y} \partial / \partial y$, and $\left|\nabla_{t} L_{i}\right|_{j}$ and $|J|_{j}$ are, respectively, the values of $\left|\nabla_{t} L_{i}\right|(i=1,2,3)$ and $|J|$ at the nodal point $j(j=1,2, \ldots, 6)$.

For the rectilinear cases, $|J|\left|\nabla L_{i}\right|=l_{i}$ with $l_{i}$ being the length of the edge facing to the vertex $i(i=1,2,3)$, and thus, the vector basis function summarized in Table II are exactly reduced to those for the rectilinear edge elements [1]-[3], [6]-[8].

Applying the finite element technique to (1), we obtain

$$
[K]\{\phi\}-\beta^{2}[M]\{\phi\}=\{0\}
$$

where $\{0\}$ is a null vector, and the global electric or magnetic field vector $\{\phi\}$ and the finite element matrices, $[K]$ and $[M]$, are given by

$$
\begin{gathered}
\{\phi\}=\left[\begin{array}{l}
\left\{\phi_{t}\right\} \\
\left\{\phi_{z}\right\}
\end{array}\right] \\
{[K]=\left[\begin{array}{cc}
{\left[K_{t t}\right]} & {[0]} \\
{[0]} & {[0]}
\end{array}\right]} \\
{[M]=\left[\begin{array}{ll}
{\left[M_{t t}\right.} & {\left[M_{t z}\right]} \\
{\left[M_{z t}\right]} & {\left[M_{z z}\right]}
\end{array}\right]}
\end{gathered}
$$

with

$$
\begin{aligned}
{\left[K_{t t}\right]=} & \sum_{e} \iint_{e}\left(k_{0}^{2} q\{U\}\{U\}^{T}+k_{0}^{2} q\{V\}\{V\}^{T}\right. \\
& -p \frac{\partial\{U\}}{\partial y} \frac{\partial\{U\}^{T}}{\partial y}-p \frac{\partial\{V\}}{\partial x} \frac{\partial\{V\}^{T}}{\partial x} \\
& \left.+p \frac{\partial\{U\}}{\partial y} \frac{\partial\{V\}^{T}}{\partial x}+p \frac{\partial\{V\}}{\partial x} \frac{\partial\{U\}^{T}}{\partial y}\right) d x, d y
\end{aligned}
$$

$$
\begin{aligned}
{\left[M_{t t}\right] } & =\sum_{e} \iint_{e}\left(p\{U\}\{U\}^{T}+p\{V\}\{V\}^{T}\right) d x d y \\
{\left[M_{t z}\right] } & =\left[M_{z t}\right]^{T} \\
& =\sum_{e} \iint_{e}\left(p\{U\} \frac{\partial\{N\}^{T}}{\partial x}+p\{V\} \frac{\partial\{N\}^{T}}{\partial y}\right) d x d y
\end{aligned}
$$

$$
\begin{aligned}
{\left[M_{z z}\right]=} & \sum_{e} \iint_{e}\left(p \frac{\partial\{N\}}{\partial x} \frac{\partial\{N\}^{T}}{\partial x}+p \frac{\partial\{N\}}{\partial y} \frac{\partial\{N\}^{T}}{\partial y}\right. \\
& \left.-k_{0}^{2} q\{N\}\{N\}^{T}\right) d x d y
\end{aligned}
$$

where [0] is a null matrix and $\sum_{e}$ extends over all different elements.

Noting (4) and Table II, the integrants in (12) to (15) are given as the functions of the local coordinates, and thus, the numerical 
TABLE III

VALUES OF WeIGHTING COEFFICIENTS AND LOCAL COORDINATES

\begin{tabular}{cccccl}
\hline$k$ & $W_{k}$ & $L_{1 k}$ & $L_{2 k}$ & $L_{3 k}$ & \\
\hline 1 & 0.225 & $a_{1}$ & $a_{1}$ & $a_{1}$ & $a_{1}=1 / 3$ \\
2 & 0.13239415 & $a_{2}$ & $a_{3}$ & $a_{3}$ & $a_{2}=0.05971587$ \\
3 & 0.13239415 & $a_{3}$ & $a_{2}$ & $a_{3}$ & $a_{3}=0.47014206$ \\
4 & 0.13239415 & $a_{3}$ & $a_{3}$ & $a_{2}$ & $a_{4}=0.79742669$ \\
5 & 0.12593918 & $a_{4}$ & $a_{5}$ & $a_{5}$ & $a_{5}=0.10128651$ \\
6 & 0.12593918 & $a_{5}$ & $a_{4}$ & $a_{5}$ & \\
7 & 0.12593918 & $a_{5}$ & $a_{5}$ & $a_{4}$ & \\
\hline
\end{tabular}

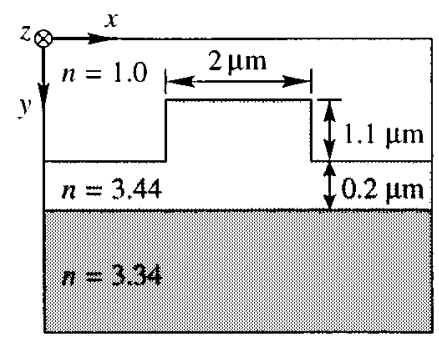

Fig. 3. Rib waveguide.

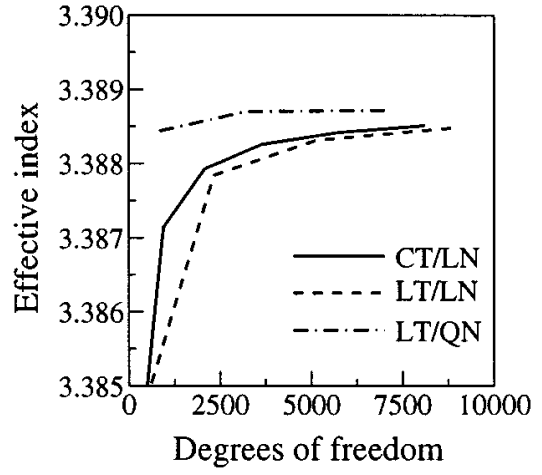

(a)

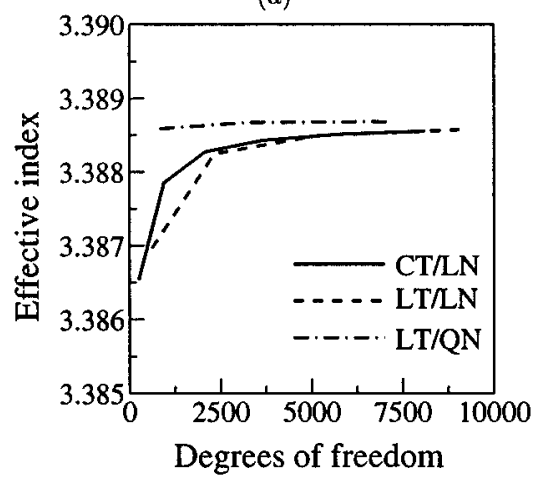

(b)

Fig. 4. Convergence of effective index for the fundamental TE-like mode in a rib waveguide calculated by using (a) the electric field and (b) the magnetic field as working variables.

integration formulas derived by Hammer et al. [9], [10] can be directly applied to (6) as follows:

$$
\begin{aligned}
\iint_{e} f(x, y) d x d y \\
=\sum_{k=1}^{7} \frac{W_{k}}{2} f\left(L_{1 k}, L_{2 k}, L_{3 k}\right)\left|J\left(L_{1 k}, L_{2 k}, L_{3 k}\right)\right|
\end{aligned}
$$

where the subscript $k$ denotes the quantity associated with the sampling point $k(k=1,2, \ldots, 7)$, and the local coordinates,

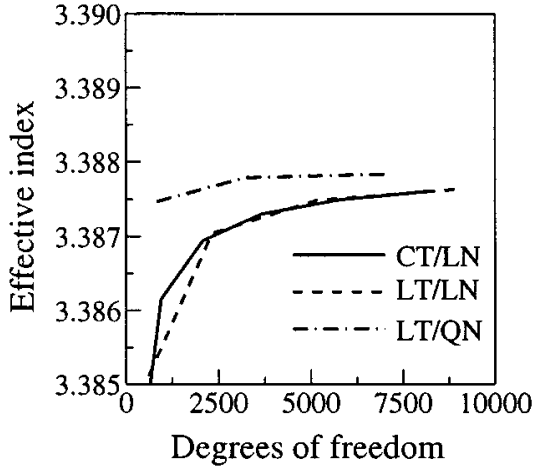

(a)

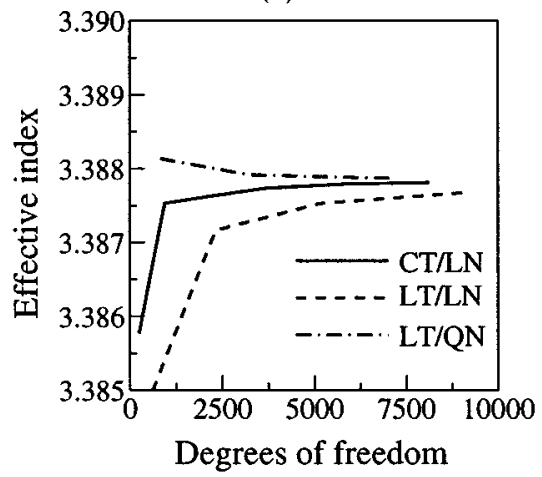

(b)

Fig. 5. Convergence of effective index for the fundamental TM-like mode int a rib waveguide calculated by using (a) the electric field and (b) the magnetic field as working variables.
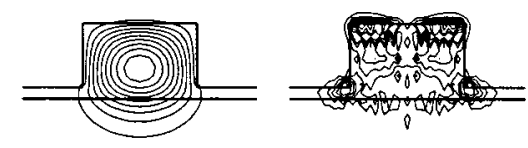

$E_{x}$

$H_{x}$
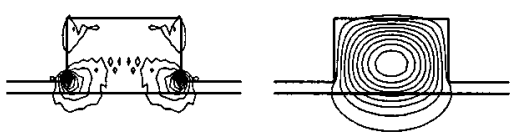

$E_{y}$

$H_{y}$
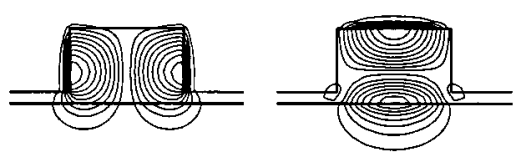

$E_{z}$

$H_{z}$

Fig. 6. Field distributions for the fundamental TE-like mode in a rib waveguide.

$L_{1 k}, L_{2 k}$, and $L_{3 k}$, are presented in Table III [9], [10]. The approximation of neglecting the second derivatives of local coordinates is very useful for evaluating the terms $\partial\{U\} / \partial y$ and $\partial\{V\} / \partial x$ included in (12).

\section{NUMERICAL RESULTS}

First, we consider a rib waveguide with straight boundaries as shown in Fig. 3. Because of the symmetry nature of the system, 

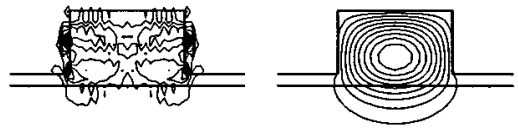

$E_{x}$
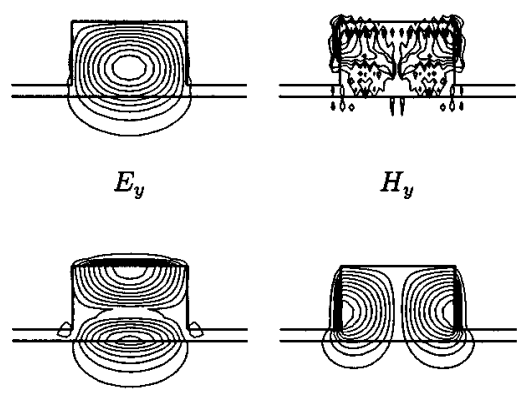

$E_{z}$

$H_{z}$

Fig. 7. Field distributions for the fundamental TM-like mode in a rib waveguide.

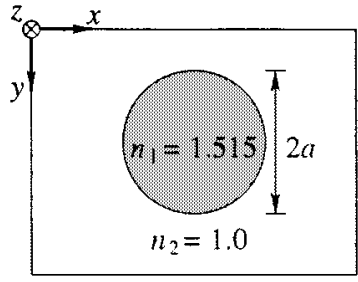

Fig. 8. Circular waveguide.

only one-half of the waveguide cross section is divided into rectilinear hybrid elements and the vector-based shape function $\mathbf{i}_{x}\{U\}+\mathbf{i}_{y}\{V\}$ and the scalar-based shape function $\{N\}$ summarized in Table II were implemented as is.

Figs. 4 and 5 show, respectively, the convergence behavior of the effective index $\beta / k_{0}$ for the fundamental transverse electric (TE)-like and transverse magnetic (TM)-like modes in the rib waveguide, where the computed results are obtained by regarding $\phi$ as $\mathbf{E}$ in (a) and as $\mathbf{H}$ in (b). The results of the LT/QN-1 and LT/QN-2 representations are almost the same each other, so the labels are rewritten as LT/QN. It is confirmed from Figs. 4 and 5 that the basis functions for the curvilinear hybrid elements are valid also for rectilinear cases and that the fastest convergence is given for the LT/QN representation. The lowest order CT/LT element works better or at least almost the same as the $\mathrm{LT} / \mathrm{LN}$ one, which is one order higher.

Figs. 6 and 7 show, respectively, the field distributions for the fundamental TE-like and TM-like modes in the rib waveguide. The electric and magnetic fields are, respectively, obtained by $\phi=\mathbf{E}$ and $\phi=\mathbf{H}$.

Next, we consider a circular waveguide with core radius $a$ and large refractive-index difference, namely, core-index $n_{1}=$ 1.515 and cladding index $n_{1}=1.0$, as shown in Fig. 8. Because of the two-fold symmetry of the system, only one-quater of the waveguide cross section is divided into curvilinear hybrid elements.

Figs. 9 and 10 show, respectively, the convergence behavior of the normalized propagation constant $b$ at the normalized frequencies $v=2$ and 5 for the fundamental mode in the cir-

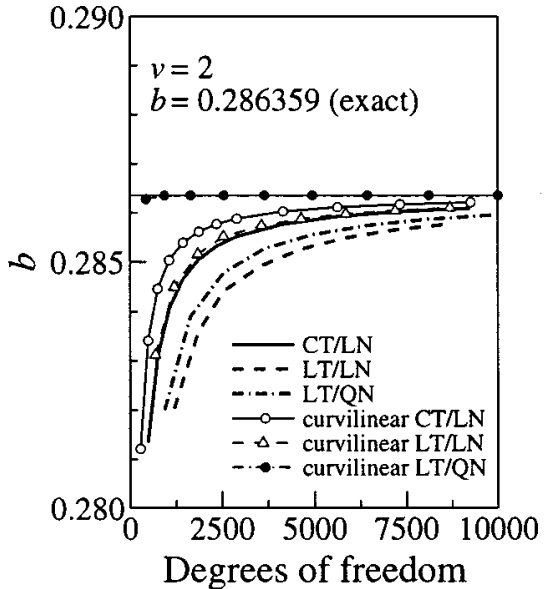

(a)

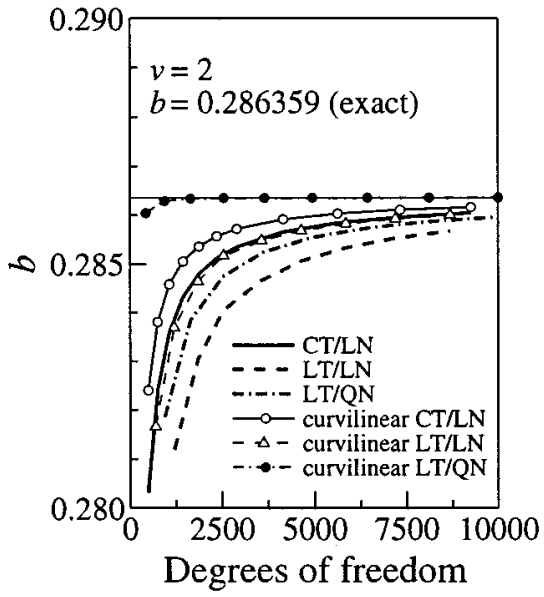

(b)

Fig. 9. Convergence of normalized propagation constant at the normalized frequency $v=2$ for the fundamental mode in a circular waveguide calculated by using (a) the electric field and (b) the magnetic field as working variables.

cular waveguide, where the computed results are obtained by regarding $\boldsymbol{\phi}$ as $\mathbf{E}$ in (a) and as $\mathbf{H}$ in (b), and $v$ and $b$ are defined as

$$
\begin{gathered}
v=k_{0} n_{1} a \sqrt{2 \Delta} \\
b=\frac{\left(\beta / k_{0}\right)^{2}-n_{2}^{2}}{n_{1}^{2}-n_{2}^{2}}
\end{gathered}
$$

with $\Delta=\left(n_{1}^{2}-n_{2}^{2}\right) /\left(2 n_{1}^{2}\right)$ being the refractive-index difference. It is confirmed from Figs. 9 and 10 that the curvilinear hybrid elements proposed here can give faster convergence than the conventional rectilinear hybrid ones and that when using the curvilinear LT/QN representation, the significantly fastest convergence is obtained, irrespective of the $v$-value, in other words, the degree of field confinement into core region. When using the conventional rectilinear elements, the lowest order CT/LN element performs better than the LT/LN one. Moreover, the CT/LN element performs even better than the LT/QN one (except in Fig. 10(a), where LT/QN works better than CT/LN, however both curves are very close). If the number of degrees of freedom is the same among CT/LN, LT/LN, and LT/QN, much more el- 


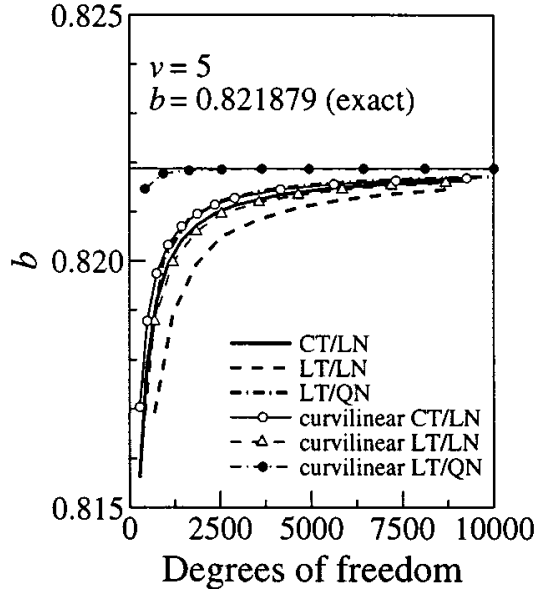

(a)

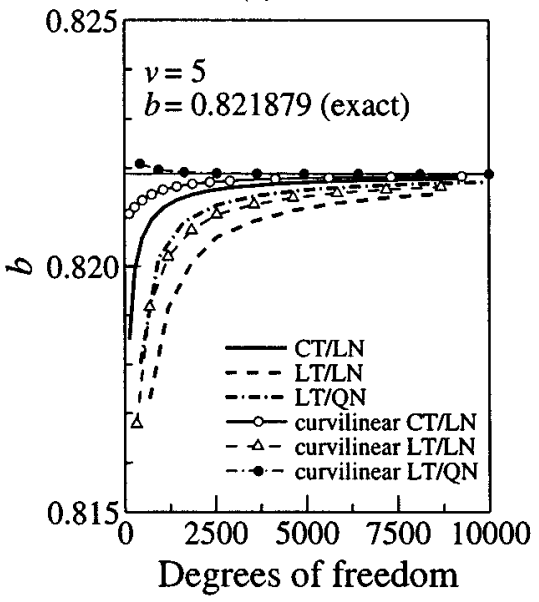

(b)

Fig. 10. Convergence of normalized propagation constant at the normalized frequency $v=5$ for the fundamental mode in a circular waveguide calculated by using (a) the electric field and (b) the magnetic field as working variables.

ements are used in CT/LN, compared to LT/LN and LT/QN. Therefore, in the rectilinear case, curved boundaries could be more accurately modeled with the lowest order CT/LN element. Anyway, from the two examples in Figs. 3 and 8, we are inclined to recommend to avoid the use of $\mathrm{LT} / \mathrm{LN}$ elements.

Figs. 11 and 12 show, respectively, the field distributions at $v=2$ and 5 for the fundamental mode in the circular waveguide. The electric and magnetic fields are, respectively, obtained by $\phi=\mathbf{E}$ and $\phi=\mathbf{H}$.

\section{CONCLUSION}

We presented a unified approach using curvilinear hybrid edge/nodal elements with triangular shape for the study of guided-wave problems. Not only the lowest order but the higher order elements were systematically constructed and were applied to rib waveguides and circular waveguides. For the guided-wave problems, we would like to recommend to use the rectilinear/curvilinear hybrid elements which are composed of the LT/QN-based edge elements and the quadratic nodal elements.

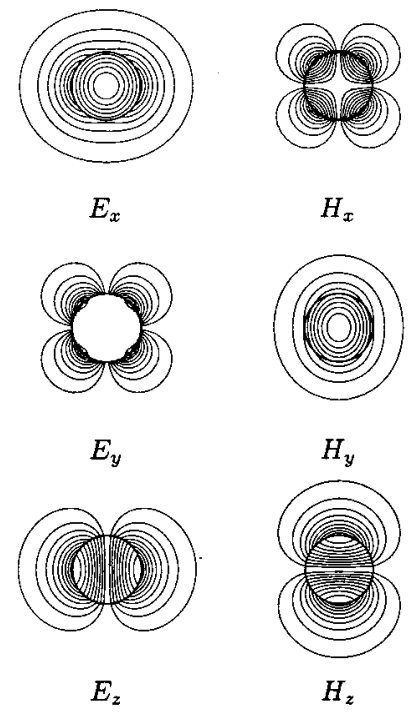

Fig. 11. Field distributions at the normalized frequency $v=2$ for the fundamental mode in a circular waveguide.

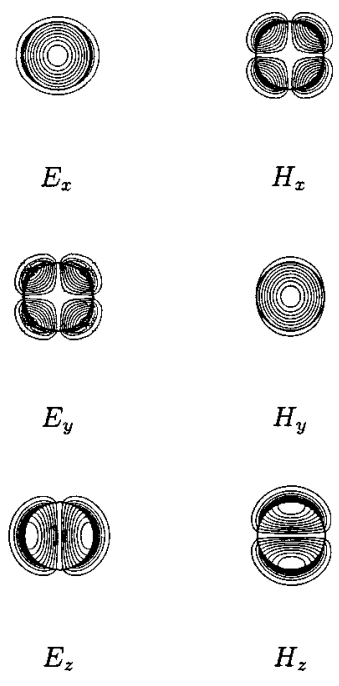

Fig. 12. Field distributions at the normalized frequency $v=5$ for the fundamental mode in a circular waveguide.

\section{REFERENCES}

[1] J.-F. Lee, D.-K. Sun, and Z. J. Cendes, "Full-wave analysis of dielectric waveguides using tangential vector finite elements," IEEE Trans. Microwave Theory Tech., vol. 39, pp. 1262-1271, Aug. 1991.

[2] M. Koshiba and K. Inoue, "Simple and efficient finite-element analysis of microwave and optical waveguides," IEEE Trans. Microwave Theory Tech., vol. 40, pp. 371-377, Feb. 1992.

[3] M. Koshiba, S. Maruyama, and K. Hirayama, "A vector finite element method with the high-order mixed-interpolation type triangular elements for optical waveguiding problems," J. Lightwave Technol., vol. 12, pp. 495-502, Mar. 1994

[4] J. S. Wang and N. Ida, "Curvilinear and higher order 'edge' finite elements in electromagnetic field computation," IEEE Trans. Magnet., vol. 29, pp. 1491-1494, Mar. 1993.

[5] G. E. Antilla and N. G. Alexopoulos, "Scattering from complex threedimensional geometries by a curvilinear hybrid finite-element-integral equation approach," J. Opt. Soc. Amer. A, vol. 11, pp. 1445-1457, Apr. 1994.

[6] J. C. Nedelec, "Mixed finite elements in R3," Numer. Math., vol. 35, pp. $315-341,1980$ 
[7] A. F. Peterson, "Vector finite element formulation for scattering from two-dimensional heterogeneous bodies," IEEE Trans. Antennas Propagat., vol. AP-43, pp. 357-365, Mar. 1994.

[8] G. Mur and A. T. Hoop, "A finite element method for computing three-dimensional electromagnetic fields in inhomogeneous media," IEEE Trans. Magnet., vol. MAG-21, pp. 2188-2191, Nov. 1985.

[9] O. C. Zienkiewitz, The Finite Element Method, 3rd ed. London, U.K. McGraw-Hill, 1977.

[10] M. Koshiba, Optical Waveguide Theory by the Finite Element Method. Tokyo, Japan: KTK Scientific/Kluwer, 1992.

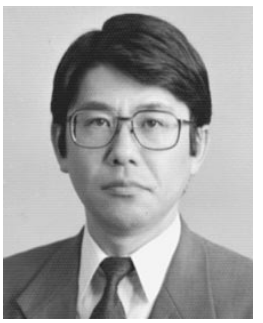

Masanori Koshiba (SM'84) was born in Sapporo, Japan, on November 23, 1948. He received the B.S., M.S., and Ph.D. degrees in electronic engineering from Hokkaido University, Sapporo, Japan, in 1971 1973, and 1976, respectively.

In 1976, he joined the Department of Electronic Engineering, Kitami Institute of Technology, Kitami, Japan. From 1979 to 1987, he was an Associate Professor of Electronic Engineering at Hokkaido University, and in 1987, he became Professor. He has been engaged in research on wave electronics, including microwaves, millimeter-waves, lightwaves, surface acoustic waves (SAW), magnetostatic waves (MSW), and electron waves, and computer-aided design and modeling of guided-wave devices using finite element method, boundary element method, beam propagation method, etc. $\mathrm{He}$ is an author or coauthor of more than 200 research papers in English and of more than 100 research papers in Japanese both in refereed journals. He authored the books Optical Waveguide Analysis (New York: McGraw-Hill) and Optical Waveguide Theory by the Finite Element Method (Tokyo/Dordrecht: KTK Scientific/Kluwer), and coauthored the books Analysis Methods for Electromagnetic Wave Problems (Norwood, MA: Artech House), Ultrafast and Ultra-parallel Optoelectronics (New York: Wiley), and Finite Element Software for Microwave Engineering (New York: Wiley).

Dr. Koshiba is a member of the Institute of Electronics, Information and Communication Engineers (IEICE), the Institute of Electrical Engineers of Japan, the Institute of Image Information and Television Engineers of Japan, the Japan Society for Simulation Technology, the Japan Society for Computational Methods in Engineering, the Japan Society of Applied Electromagnetics and Mechanics, the Japan Society for Computational Engineering and Science, and the Applied Computational Electromagnetics Society (ACES). In 1987, 1997, and 1999, he recieved the Excellent Paper Awards from the IEICE, and in 1998, he recieved the Electronics-Society Award from the IEICE.

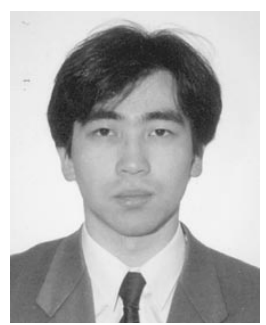

Yasuhide Tsuji (M'97) was born in Takikawa, Japan, on December 31, 1967. He received the B.S., M.S., and Ph.D. degrees in electronic engineering from Hokkaido University, Sapporo, Japan, in 1991, 1993, and 1996, respectively.

In 1996, he joined the Department of Applied Electronic Engineering, Hokkaido Institute of Technology, Sapporo, Japan. Since 1997, he has been an Associate Professor of Hokkaido University, Sapporo, Japan. He has been engaged in research on wave electronics.

Dr. Tsuji is a member of the Institute of Electronics, Information and Communication Engineers (IEICE). In 1997 and 1999, he recieved the Excellent Pape Awards from the IEICE, and in 1999, he recieved the Young Scientist Award from the IEICE. 